\title{
Global Existence and Exponential Stability of Small Solutions to Nonlinear Viscoelasticity
}

\author{
S. Kawashima ${ }^{1}$ and Y. Shibata ${ }^{2}$ \\ ${ }^{1}$ Department of Applied Science, Faculty of Engineering 36, Kyushu University, Fukuoka 812, \\ Japan \\ 2 Institute of Mathematics, University of Tsukuba, Tsukuba-shi, Ibaraki 305, Japan \\ Received October 25, 1991
}

\begin{abstract}
The global existence of smooth solutions to the equations of nonlinear hyperbolic system of 2nd order with third order viscosity is shown for small and smooth initial data in a bounded domain of $n$-dimensional Euclidean space with smooth boundary. Dirichlet boundary condition is studied and the asymptotic behaviour of exponential decay type of solutions as $t$ tending to $\infty$ is described. Time periodic solutions are also studied. As an application of our main theorem, nonlinear viscoelasticity, strongly damped nonlinear wave equation and acoustic wave equation in viscous conducting fluid are treated.
\end{abstract}

\section{Introduction}

In this paper, we are concerned with the global existence and exponential stability of small and smooth solutions to the following equations:

$$
\begin{array}{rlrl}
A_{0}(U) \partial_{t}^{2} u+A_{j}(U) \partial_{j} \partial_{t} u-A_{i j}(U) \partial_{i} \partial_{j} u & \\
-B_{i j}(U) \partial_{i} \partial_{j} \partial_{t} u & =f & \text { in }[0, \infty) \times \Omega, \\
u & =0 & \text { on }[0, \infty) \times \partial \Omega, \\
u(0, x)=u_{0}(x) \text { and } \quad & u_{t}(0, x)=u_{1}(x) & \text { in } \Omega .
\end{array}
$$

The existence of time periodic solutions is also studied. Here, $\Omega$ is a bounded domain in $\mathbb{R}^{n}$ with $C^{\infty}$ boundary $\partial \Omega, U=\left(\nabla u, u_{t}, \nabla u_{t}\right), u_{t}=\partial_{t} u=\partial u / \partial t$, $\nabla u=\left(\partial_{1} u, \ldots, \partial_{n} u\right), \quad \partial_{j} u=\partial u / \partial x_{j} \quad(j=1, \ldots, n), \quad x=\left(x_{1}, \ldots, x_{n}\right) \in \mathbb{R}^{n}$, $u={ }^{t}\left(u_{1}, \ldots, u_{d}\right)$ is a $d$-vector of real-valued functions $\left({ }^{t} M\right.$ means the transposed $M)$, and the summation convention is understood where the indices run through 1 to $n$. The $A_{0}(U), A_{j}(U), A_{i j}(U)$ and $B_{i j}(U)$ are $d \times d$ matrices of real-valued functions defined on $\left\{U \in \mathbb{R}^{(2 n+1) d}|| U \mid \leqq K\right\}$ and in $C^{\infty}$ there, which satisfies the following assumption: 
Assumption 1.1. (1) ${ }^{t} A_{0}(U)=A_{0}(U),{ }^{t} A_{j}(U)=A_{j}(U),{ }^{t} A_{i j}(U)=A_{j i}(U)$ and ${ }^{t} B_{i j}(U)=B_{j i}(U)$,

$$
\begin{gathered}
A_{0}(0) \geqq \alpha_{0} I_{d}, \\
M_{i j}(0) \xi_{i} \xi_{j} \geqq \alpha_{0}|\xi|^{2} I_{d} \text { for any } \xi=\left(\xi_{1}, \ldots, \xi_{n}\right) \in \mathbb{R}^{n},
\end{gathered}
$$

with some constant $\alpha_{0}>0$, where $M=A$ and $B$, and $I_{d}$ is the $d \times d$ identity matrix.

From Assumption 1.1, Eq. (1.1) is a nonlinear hyperbolic system of $2^{\text {nd }}$ order with nonlinear viscosity term: $B_{i j} \partial_{i} \partial_{j} \partial_{t} u$ and it appears in models of nonlinear viscoelasticity, acoustic wave equation in a viscous conducting fluid, strongly damped wave equations and so on. Concerning global existence of solutions, the problem (1.1)-(1.3) was first studied in one space dimension and scalar case, and classical solutions for arbitrary smooth data, their asymptotic properties and so on were found in $[1,2,8,9,14,15,20$ and 25]. Concerning the strongly damped nonlinear scalar wave equation of the form: $u_{t t}-\operatorname{div} \sigma(\nabla u)-\Delta u_{t}=0$, Pecher [19] proved the global existence of classical solutions for arbitrary data in the two dimensional Cauchy problem case $\left(\Omega=\mathbb{R}^{2}\right)$ under some growth order condition on $\sigma^{\prime}$ (see also [7]), and global weak solutions was studied by J. Clemént [6]. The semilinear case: $u_{t t}-a \Delta u-\Delta u_{t}+f\left(u, \nabla u, u_{t}, \nabla u_{t}\right)=0(a \geqq 0)$, was studied by [3, $4,5,19$ and 24]. The global strong solution (not so smooth, e.g., $\nabla u, \nabla u_{t}$ are Hölder continuous) was studied by [12 and 13] under some growth order condition on $A_{i j}(U)$, in the case where $A_{j}(U)=0(j=1, \ldots, n)$ and $A_{0}(U)=I_{d}$.

In the treatment of the above studies for the higher dimensional case, it plays an essential role the estimation of $L^{p}$-norm of solutions to the linear parabolic part: $\left(\partial_{t}-B_{i j}(0) \partial_{i} \partial_{j}\right) u_{t}$, and the nonlinear term was restricted strongly, because the estimation of derivatives of higher order was not enough.

In order to study more general equations containing important physical models, one approach is to abandon arbitrariness of data and to look for small solutions. In this direction, the $L^{2}$ framework is better, because the equation is hyperbolic with a strong damping term and a good estimate of derivatives with respect to both $t$ and $x$ are expected thanks to the hyperbolicity. In fact, Y. Ebihara $[10,11]$ studied the small and smooth solution to the strongly damped scalar wave equation of the form: $u_{t t}-\Delta u_{t}=f\left(u, \nabla u, \partial_{x}^{2} u, u_{t}, \nabla u_{t}\right), \partial_{x}^{2} u=\left(\partial_{x}^{\alpha} u,|\alpha|=2\right)$ with suitable hyperbolic assumption, by using the so-called $\stackrel{\circ}{H}^{k}$-Galerkin method. Mizohata and Ukai [18] studied also small and smooth solutions in the $L^{2}$ framework to acoustic wave equation in a viscous conducting fluid described by:

$$
u_{t t}-a \Delta u-b \Delta u_{t}=c\left(|\nabla u|^{2}+\left|u_{t}\right|^{2}\right)_{t}
$$

with some constants $a>0, b>0$ and $c \neq 0$. In these studies, to these authors it does not seem that they obtained enough estimates of higher order derivatives, and then the class of solutions in [10 and 11] was restricted and the proof in [18] depended deeply on the special form of the nonlinear term.

Our purpose is to prove the unique existence theorem of global small and smooth solutions to the problem (1.1)-(1.3) in the $L^{2}$ framework, studying an exponential decay estimate of solutions. The crucial point is to get the estimate of higher order derivatives with respect to both $t$ and $x$. If the viscosity term: $B_{i j} \partial_{i} \partial_{j} \partial_{t} u$ disappears, the estimate with respect to $x$ are usually obtained by using the ellipticity of the terms: $A_{i j} \partial_{i} \partial_{j} u$. However, in our case, this method does not work, because the two terms: $A_{i j} \partial_{i} \partial_{j} u$ and $B_{i j} \partial_{i} \partial_{j} \partial_{t} u$ must be evaluated at the same 
time. The main difficulty comes from this point and our technical contribution is mainly how to derive the estimate of spatial derivatives, which seems a new point compared with previous works.

Finally, we would like to remark that more general equation (1.1) with $f=F\left(t, x, \bar{\partial}^{2} u, \partial_{x}^{2} u_{t}\right)\left(\bar{\partial}^{2} u=\left(\partial_{x}^{\alpha} \partial_{t}^{j} u ;|\alpha|+j \leqq 2\right)\right)$ can be handled with under a suitable assumption on $F$ which guarantees the strict hyperbolicity and the essential appearance of a viscosity term, by combining a technique here of obtaining the exponential decay estimate with a technique due to [23] of reducing a fully nonlinear hyperbolic operator to some quasilinear hyperbolic-elliptic coupled system (see also [17]). However, to deal with the fully nonlinear case at least our essential arguments concerning the estimate of spatial derivatives are not easy to understand in this paper. Therefore, we shall treat (1.1) only. We believe that Eq. (1.1) under Assumption 1.1 contains plenty of important physical models.

\section{Statement of Main Results and Examples}

First, we explain our basic notation. Let $L^{2},\left(\right.$, ) and $\|\cdot\|$ denote the usual $L^{2}$ space on $\Omega$, its inner-product and its norm, respectively. Put

$$
\begin{aligned}
\partial_{y}^{k} u & =\left(\partial_{y}^{\alpha} u,|\alpha|=k\right), \quad \bar{\partial}_{y}^{k} u=\left(\partial_{y}^{j} u, 0 \leqq j \leqq k\right) \quad(y=x \text { and } t), \\
\partial^{k} u & =\left(\partial_{x}^{\alpha} \partial_{t}^{j} u,|\alpha|+j=k\right), \quad \bar{\partial}^{k} u=\left(\partial^{j} u, 0 \leqq j \leqq k\right), \\
H^{k} & =\left\{u \in L^{2} \mid \partial_{x}^{\alpha} u \in L^{2} \text { for }|\alpha| \leqq k\right\}, \quad H_{0}^{1}=\left\{u \in H^{1} \mid u=0 \text { on } \partial \Omega\right\}, \\
(u, v)_{k} & =\sum_{|\alpha| \leqq k}\left(\partial_{x}^{\alpha} u, \partial_{x}^{\alpha} v\right), \quad\|u\|_{k}=(u, u)_{k}^{1 / 2}, \quad\|u\|_{\infty}=\sup _{x \in \Omega}|u(x)| .
\end{aligned}
$$

For the time interval $I \subset \mathbb{R}$ and a Banach space $B, C^{k}(I, B)$ is the set of all $B$-valued continuous functions which are continuously $k$ times differentiable in $t \in I$. Put

$$
E_{N}([S, T])=\bigcap_{j=0}^{N-1} C^{j+1}\left([S, T] ; H^{N-j}\right) .
$$

For a $d$-vector $v$ of functions in $(t, x)$, the capital letter $V$ means that $V=\left(\nabla v, v_{t}, \nabla v_{t}\right)$. Put

$$
\begin{aligned}
P(v) u & =A_{0}(V) \partial_{t}^{2} u+A_{j}(V) \partial_{j} \partial_{t} u-A_{i j}(V) \partial_{i} \partial_{j} u-B_{i j}(V) \partial_{i} \partial_{j} \partial_{t} u, \\
p_{v}(t) & =\left\|\bar{\partial}^{[n / 2]+2} V(t, \cdot)\right\|, \quad N_{0}=[n / 2]+3,
\end{aligned}
$$

where $[r]$ is the largest integer $\leqq r$. For two operators $A$ and $B$, the commutator of $A$ and $B$ is denoted by: $[A, B]=A B-B A$. In order to estimate $\|u\|$, we always use Poincaré's inequality: $\|u\| \leqq \alpha_{1}\|\nabla u\|$ for $u \in H_{0}^{1}$, where $\alpha_{1}$ is a suitable constant. From Sobolev's inequality: $\|u\|_{\infty} \leqq \alpha_{2}\|u\|_{N_{0}-2}$, it follows that

$$
\left\|\bar{\partial}^{1} V(t, \cdot)\right\|_{\infty} \leqq \alpha_{2} p_{v}(t) .
$$

From Poincaré's inequality, it follows that what $u \in E_{N}([S, T])$ is equivalent to that

$$
U \in \bigcap_{j=0}^{N-1} C^{j}\left([S, T] ; H^{N-1-j}\right)
$$

provided that $u(t, \cdot)=0$ on $\partial \Omega$ for $t \in[S, T]$. Since $C_{0}^{\infty}(\Omega)=\left\{u \in C^{\infty}\left(\mathbb{R}^{n}\right) \mid\right.$ supp $u \subset \Omega\}$ is dense in $H_{0}^{1}$, using Assumption 1.1-(3) and Parseval's formula for 
Fourier transform in $\mathbb{R}^{n}$, we have

$$
\left(M_{i j}(0) \partial_{j} u, \partial_{i} u\right) \geqq \alpha_{0}\|\nabla u\|^{2} \text { for } u \in H_{0}^{1} \text { and } M=A \text { and } B \text {. }
$$

In view of this and Assumption 1.1-(2), we choose $K$ so small that

$$
\left(\alpha_{0} / 2\right)\|\nabla u\|^{2} \leqq\left(M_{i j}(V) \partial_{j} u, \partial_{i} u\right) \leqq \alpha_{3}\|\nabla u\|^{2} \text { for any } u \in H_{0}^{1} \text { and } M=A \text { and } B \text {, }
$$

$$
\left(\alpha_{0} / 2\right) I_{d} \leqq A_{0}(V) \leqq \alpha_{3} I_{d},
$$

provided that $|V| \leqq K$. Let $\alpha_{4}>0$ be a constant such that $\left|\left(A_{i j}(V), B_{i j}(V), A_{j}(V)\right)\right|$ $\leqq \alpha_{4}$ for any $|V| \leqq K$. The letter $C$ denotes various constants depending only on $\alpha_{0}, \alpha_{1}, \alpha_{2}, \alpha_{3}, \alpha_{4}, K, n$ and $\Omega$ essentially. We denote also various constants depending on quantities $A, B$ and so on by $C(A, B, \ldots)$.

Now, we shall explain compatibility condition. If $u$ satisfies (1.1) and the condition: $|U| \leqq K$, differentiation of (1.1) with respect to $t$ implies that $\partial_{t}^{k} u(k \geqq 2)$ are given successively by the formula:

$$
\begin{aligned}
\partial_{t}^{k} u(t, \cdot) & =A_{0}(U(t, \cdot))^{-1}\left\{A_{i j}(U(t, \cdot)) \partial_{i} \partial_{j} \partial_{t}^{k-2} u(t, \cdot)+B_{i j}(U(t, \cdot)) \partial_{i} \partial_{j} \partial_{t}^{k-1} u(t, \cdot)\right. \\
& \left.-A_{j}(U(t, \cdot)) \partial_{j} \partial_{t}^{k-1} u(t, \cdot)+\left[\partial_{t}^{k-2}, P(u)\right] u(t, \cdot)+\partial_{t}^{k-2} f(t, \cdot)\right\}
\end{aligned}
$$

Using the formula (2.6), from $u_{0}$ and $u_{1}$ we can define successively the value of $\partial_{t}^{k} u$ at $t=0$, which is denoted by $u_{k}$. What $u=0$ on $\partial \Omega$ implies that $u_{k}=0$ on $\partial \Omega$, which is the compatibility condition at $t=0$ and the boundary.

The assumption on $u_{0}, u_{1}$ and $f$ is the following.

Assumption 2.1. Let $N$ be an integer $\geqq N_{0}$. Suppose:

$$
\begin{gathered}
u_{0} \in H^{N} \cap H_{0}^{1}, \quad u_{j+1} \in H^{N-j} \cap H_{0}^{1}(0 \leqq j \leqq N-1), \\
f \in \bigcap_{j=0}^{N-2} C^{j}\left([0, \infty) ; H^{N-2-j}\right) \cap C^{N-1}\left([0, \infty) ; L^{2}\right), \\
\lambda_{N}(\infty)=\sup _{t>0} e^{\mu t}\left\{\left\|\bar{\partial}^{N-2} f(t, \cdot)\right\|+\left\|\partial_{t}^{N-1} f(t, \cdot)\right\|\right)<\infty
\end{gathered}
$$

for some $\mu \geqq 0$.

The following theorem is our main result.

Theorem 2.2. Suppose that Assumptions 1.1 and 2.1 are satisfied. Put

$$
\rho_{k}=\sum_{j=0}^{k}\left\|u_{j}\right\|_{k-j}+\left\|\nabla u_{k}\right\|, \quad \tilde{\rho}_{k}=\left\|u_{0}\right\|_{k}+\sum_{j=0}^{k-1}\left\|u_{j+1}\right\|_{k-j} .
$$

(A) Then, there exists an $\varepsilon>0$ independent of $u_{0}, u_{1}, f$ and $N$ such that if $\tilde{\rho}_{N_{0}}+\lambda_{N_{0}}(\infty) \leqq \varepsilon$, then the problem $(1.1)-(1.3)$ admits a unique solution $u \in$ $E_{N}([0, \infty))$ satisfying the condition: $\|U(t, \cdot)\|_{\infty} \leqq K / 2$ for all $t \in[0, \infty)$.

(B) Moreover, the following asymptotic behavior holds. (1) If $\mu=0$, then there exists an $\varepsilon_{N}>0$ depending on $N$ such that if $\lambda_{N_{0}}(\infty) \leqq \varepsilon_{N}$, then

$$
\left\|\bar{\partial}^{k-1} U(t, \cdot)\right\| \leqq e^{-\omega t} \phi_{k}\left(\rho_{k}\right)+\psi_{k}\left(\lambda_{k}(\infty)\right)
$$


for any $N_{0} \leqq k \leqq N$, where $\omega$ is some positive constant and $\phi_{k}(t)=\psi_{k}(t)=$ $C(k)(1+t)^{m_{k}} t$ for some integer $m_{k} \geqq 0$ depending on $k$.

(2) If $\mu>0$, then

$$
\left\|\bar{\partial}^{k-1} U(t, \cdot)\right\| \leqq e^{-\omega t} \phi_{k}\left(\rho_{k}\right)+e^{-\gamma t} \psi_{k}\left(\lambda_{k}(\infty)\right)
$$

for any $N_{0} \leqq k \leqq N$ and $0<\gamma<\min (\mu, \omega)$, where $\phi_{k}(t)=C(k)(1+t)^{m_{k}} t$ and $\psi_{k}(t)=C(k, \gamma)(1+t)^{m_{k}} t$.

By using the technique due to P. Rabinowitz [21] and A. Matsumura [17], exploiting the exponential stability (2.8), we obtain the existence of global small periodic solutions provided that $f$ is periodic with respect to $t$.

Theorem 2.3. Suppose that Assumption 1.1 is satisfied and that

$$
\begin{gathered}
f \in \bigcap_{j=0}^{N-2} C^{j}\left(\mathbb{R} ; H^{N-2-j}\right) \cap C^{N-1}\left(\mathbb{R} ; L^{2}\right) \quad\left(N \geqq N_{0}\right), \\
f\left(t+T_{0}, x\right)=f(t, x) \quad \text { for any }(t, x) \in \mathbb{R} \times \Omega .
\end{gathered}
$$

(A) (Existence of periodic solution). Then, there exists an $\varepsilon_{N}^{\prime}>0$ depending on $N$ essentially such that if $\lambda_{N_{0}}(\infty) \leqq \varepsilon_{N}^{\prime}$, then the problem (1.1) and (1.2) admits a periodic solution $u \in E_{N}(\mathbb{R})$ satisfying the conditions: $\|U(t, \cdot)\|_{\infty} \leqq K$ for all $t \in \mathbb{R}$ and $u\left(t+T_{0}, x\right)=u(t, x)$ for any $(t, x) \in \mathbb{R} \times \Omega$.

(B) (Uniqueness of periodic solution). Let $u, v \in E_{N_{0}}(\mathbb{R})$ be two periodic solutions with period $T_{0}$ to the problem (1.1) and (1.2). Then, there exists an $\varepsilon^{\prime \prime}>0$ independent of $u$ and $v$ such that $u=v$ provided that $p_{u}(t), p_{v}(t) \leqq \varepsilon^{\prime \prime}$ for any $t \in \mathbb{R}$.

(C) (Exponential stability of periodic solution). There exists an $\varepsilon_{N}^{\prime \prime}>0$ depending on $N$ such that for the time periodic solution $u$ obtained in (A), any solution $v$ to the problem (1.1)-(1.3) satisfying the following:

$$
v \in E_{N}([0, \infty)) \text { and }\left\|\bar{\partial}_{t}^{N} v(0, \cdot)\right\| \leqq \varepsilon_{N}^{\prime \prime},
$$

is asymptotic to $u$ exponentially as $t \rightarrow \infty$, that is,

$$
\left\|\bar{\partial}^{N-2}(U-V)(t, \cdot)\right\| \leqq C\left(N, \lambda_{N}(\infty)\right) e^{-\omega t}\left\|\bar{\partial}^{N-2}(U-V)(0, \cdot)\right\|
$$

for all $t>0$ with suitable $\omega>0$.

Now, we shall discuss some examples.

Example 2.4. The so-called strongly damped nonlinear wave equation is described by:

$$
\partial_{t}^{2} u-\partial_{j}\left(a_{j}(\nabla u)\right)-\lambda \Delta u_{t}=f,
$$

where $u, a_{j}$ and $f$ are scalar valued functions. If $\lambda>0$ and

$$
\left(\partial a_{i} / \partial u_{j}\right)(0) \xi_{i} \xi_{j} \geqq \alpha_{0}|\xi|^{2} \text { for any } \xi \in \mathbb{R}^{n} \text {, where } u_{j}=\partial_{j} u,
$$

then Assumption 1.1 is satisfied with $d=1, A_{0}=1, A_{j}=0 \quad(j=1, \ldots, n)$, $A_{i j}=\partial a_{i} / \partial u_{j}$ and $B_{i j}=\lambda \delta_{i j}, \delta_{i j}$ being the Kronecker's delta symbol.

Example 2.5. The acoustic wave equation in viscous conducting fluid (1.4) is described by (1.1) with $d=1, A_{0}=1-2 c \partial_{t} u, A_{j}=-2 c \partial_{j} u, A_{i j}=a \delta_{i j}$ and $B_{i j}=b \delta_{i j}$. Assumption 1.1 is obviously satisfied. 
Example 2.6. The motion of viscoelastic material (cf. [20, pp. 56 and 57]): Let a viscoelastic material occupy $\Omega$ in an equilibrium state. Let $x$ denote the coordinates of the material points, $\rho(x)$ the density at $x$ in the equilibrium state, $u(t, x)$ the displacement vector from the equilibrium configuration, $\gamma=\nabla u=$ the $n \times n$ matrix $\left(\partial_{i} u_{j}\right)$ the Green-Lagrange strain tensor, $S$ the Piola-Kirchhoff stress tensor and $w(\gamma)$ the free energy function which is assumed to be a function of strain only. The constitutive law of Kelvin-Voigt viscoelasticity reads:

$$
S=\frac{\partial w}{\partial \gamma}(\gamma)+T(\gamma, \dot{\gamma}) \quad\left(\dot{\gamma}=\gamma_{t}\right)
$$

where $T(\gamma, \dot{\gamma})=\left(T_{i j}\right)$ is a $n \times n$ matrix of nonlinear functions representing the viscoelastic part of the stress. The $T$ vanishes if the strain is independent of time, that is, $T(\gamma, 0)=0$. The balance law of linear momentum reads:

$$
\rho(x) \partial_{t}^{2} u-\operatorname{div} S=f \text { in } \Omega
$$

for any $t>0$. Suppose that $\rho(x)=\rho>0$ constant and that the displacement is prescribed at the boundary, that is, $u=0$ on $\partial \Omega$ for any $t>0$. Calculating the divergence part in (2.11), we can rewrite (2.11) as follows:

$$
\rho \partial_{t}^{2} u-A_{i j} \partial_{i} \partial_{j} u-B_{i j} \partial_{i} \partial_{j} \partial_{t} u=f \text { in } \Omega \text {. }
$$

Therefore, the motion of viscoelastic material is described by (1.1) and (1.2), setting $A_{i j}=$ the $n \times n$ matrix $\left(a_{k i l j}\right)\left(a_{k i l j}=\partial^{2} w / \partial \gamma_{k i} \partial \gamma_{l j}+\partial T_{k i} / \partial \gamma_{l j}, \gamma_{l j}=\partial_{j} u_{l}\right), B_{i j}=$ the $n \times n$ matrix $\left(b_{k i l j}\right)\left(b_{k i l j}=\partial T_{k i} / \partial \dot{\gamma}_{l j}, \dot{\gamma}_{l j}=\partial_{j} \partial_{t} u_{l}\right), A_{0}=\rho I_{n}$ and $A_{j}=0(j=1, \ldots$, $n)$. If $\partial T_{k i} / \partial \gamma_{l j}=\partial T_{l j} / \partial \gamma_{k i}$ and $\partial T_{k i} / \partial \dot{\gamma}_{l j}=\partial T_{l j} / \partial \dot{\gamma}_{k i}$, then we have

$$
{ }^{t} A_{i j}=A_{j i} \text { and }{ }^{t} B_{i j}=B_{j i} .
$$

Since $T(\gamma, 0)=0$, we have

$$
a_{k i l j}(0)=\left(\partial^{2} w / \partial \gamma_{k i} \partial \gamma_{l j}\right)(0) \text { and } b_{k i l j}(0)=\left(\partial T_{k i} / \partial \dot{\gamma}_{l j}\right)(0,0) \text {. }
$$

In many models appearing in the theory of elasticity, we may assume that

$$
m_{k i l j}(0) \eta_{k} \eta_{l} \xi_{i} \xi_{j} \geqq \alpha_{0}|\xi|^{2}|\eta|^{2} \text { for any } \xi, \eta \in \mathbb{R}^{n}
$$

where $m=a$ and $b$, which implies that Assumption 1.1-(3) is satisfied in the present case.

\section{Preparation for Later Sections}

As a preparation for our a priori estimate of solutions locally in time, in this section we discuss some estimate of composite function and multiplication of function in paragraph 3.1, the first energy inequality of exponential decay type in paragraph 3.2, and the estimate for spatial derivatives in paragraphs 3.3 and 3.4. In particular, the idea of paragraph 3.3 is new and one of our contributions to this field. Below, $\omega$ and $\beta_{j}(j=1,2, \ldots)$ denote some special positive constants.

3.1. Estimates of Composite Function and Multiplication of Functions. All the estimates here are derived easily by using the following well-known estimate:

$$
\|f \cdot g\| \leqq C(a, b)\|f\|_{a}\|g\|_{b} \quad \text { for } a, b \geqq 0, \text { and } a+b>\frac{n}{2} .
$$

So we may state all the assertions without any proof. 
Lemma 3.1.1. Suppose that $F(v)$ is a function defined for $|v| \leqq K$, which is in $C^{\infty}$ there and satisfies the condition: $F(0)=0$. If $k>n / 2$ and $\|v(t, \cdot)\|_{\infty} \leqq K$, then

$$
\left\|\bar{\partial}^{k} F(v(t, \cdot))\right\| \leqq C(k, F)\left(1+\left\|\bar{\partial}^{k} v(t, \cdot)\right\|\right)^{k-1}\left\|\bar{\partial}^{k} v(t, \cdot)\right\| .
$$

Moreover, if $k \geqq[n / 2]+3$ and $\|v(t, \cdot)\|_{\infty} \leqq K$, then

$$
\begin{aligned}
\left\|\bar{\partial}^{k} F(v(t, \cdot))\right\| \leqq & \left\|F^{\prime}(v(t, \cdot))\right\|_{\infty}\left\|\partial^{k} v(t, \cdot)\right\| \\
& +C(k, F)\left(1+\left\|\bar{\partial}^{k-1} v(t, \cdot)\right\|\right)^{k-1}\left\|\bar{\partial}^{k-1} v(t, \cdot)\right\| .
\end{aligned}
$$

Lemma 3.1.2. (1)

$$
\begin{aligned}
& \left\|\bar{\partial}^{1}\left(\partial^{k}(u(t, \cdot) v(t, \cdot))-u(t, \cdot) \partial^{k} v(t, \cdot)\right)\right\| \\
& \quad \leqq\left\{\begin{array}{cc}
C(k)\left\|\bar{\partial}^{[n / 2]+2} u(t, \cdot)\right\|\left\|\bar{\partial}^{k} v(t, \cdot)\right\| \quad \text { for } 0 \leqq k \leqq[n / 2]+1, \\
C(k)\left\{\left\|\bar{\partial}^{k+1} u(t, \cdot)\right\|\left\|\bar{\partial}^{[n / 2]+1} v(t, \cdot)\right\|+\left\|\bar{\partial}^{[n / 2]+2} u(t, \cdot)\right\|\left\|\bar{\partial}^{k} v(t, \cdot)\right\|\right. \\
\left.+\left\|\bar{\partial}^{k} u(t, \cdot)\right\|\left\|\bar{\partial}^{k-1} v(t, \cdot)\right\|\right\} \quad \text { for } k \geqq[n / 2]+2 .
\end{array}\right.
\end{aligned}
$$

(2) Let $0 \leqq l \leqq k$. Then,

$$
\begin{aligned}
& \left\|\partial^{l}\left(\partial^{k-l}(u(t, \cdot) v(t, \cdot))-u(t, \cdot) \partial^{k-l} v(t, \cdot)\right)\right\| \\
& \quad \leqq \begin{cases}C(k)\left\|\bar{\partial}^{[n / 2]+2} u(t, \cdot)\right\|\left\|\bar{\partial}^{k-1} v(t, \cdot)\right\| & \text { for } 1 \leqq k \leqq[n / 2]+1, \\
C(k)\left\|\bar{\partial}^{k} u(t, \cdot)\right\|\left\|\bar{\partial}^{k-1} v(t, \cdot)\right\| & \text { for } k \geqq[n / 2]+2 .\end{cases}
\end{aligned}
$$

\subsection{First Energy Inequality.}

Theorem 3.2.1. Suppose that Assumption 1.1 is satisfied. Let $u$ and $v$ be two vectors of functions satisfying:

$$
\begin{gathered}
u \in E_{2}([S, T]), \quad v \in E_{N}([S, T]), \\
\|V(t, \cdot)\|_{\infty} \leqq K \text { for } t \in[S, T], u=0 \text { on }[S, T] \times \partial \Omega .
\end{gathered}
$$

Then, there exist positive numbers $\omega, \beta_{1}$ and $\beta_{2}$ such that if $p_{v}(t) \leqq \beta_{1}$ for $t \in[S, T]$, then

$$
\begin{aligned}
& \Psi(\|U(t, \cdot)\|, \omega, S)+\int_{S}^{t} e^{2 \omega s}\left\|u_{t t}(s, \cdot)\right\|^{2} d s \\
& \quad \leqq \beta_{2}\left\{e^{2 \omega S}\|U(S, \cdot)\|^{2}+\int_{S}^{t} e^{2 \omega s}\|P(v) u(s, \cdot)\|^{2} d s \quad \text { for } t \in[S, T] .\right.
\end{aligned}
$$

Here and hereafter, we put

$$
\Psi(l(t), \omega, S)=e^{2 \omega t} l(t)^{2}+\int_{S}^{t} e^{2 \omega s} l(s)^{2} d s .
$$


Proof. Let $\delta$ be a constant $\in(0,1)$ determined later and put

$$
\begin{aligned}
E= & \left(A_{0} u_{t}, u_{t}\right)+\left(\left(A_{i j}+\delta B_{i j}\right) \partial_{j} u, \partial_{i} u\right)+\delta^{2}\left(B_{i j} \partial_{j} u_{t}, \partial_{i} u_{t}\right)+2 \delta\left(A_{0} u_{t}, u\right) \\
& +2 \delta^{2}\left(A_{i j} \partial_{j} u, \partial_{i} u_{t}\right), \\
F= & \left(B_{i j} \partial_{j} u_{t}, \partial_{i} u_{t}\right)+\delta\left(A_{i j} \partial_{j} u, \partial_{i} u\right)+\delta^{2}\left(A_{0} u_{t t}, u_{t t}\right)-\delta\left(A_{0} u_{t}, u_{t}\right) \\
& +\delta\left(A_{j} \partial_{j} u_{t}, u\right)+\delta^{2}\left(A_{j} \partial_{j} u_{t}, u_{t t}\right)-\delta^{2}\left(A_{i j} \partial_{j} u_{t}, \partial_{i} u_{t}\right), \\
R= & \frac{1}{2}\left(\left(\left(A_{0}\right)_{t}+\partial_{j} A_{j}\right) u_{t}, u_{t}\right)+\delta\left(\left(A_{0}\right)_{t} u_{t}, u\right)+\frac{1}{2}\left(\left(A_{i j}+\delta B_{i j}\right)_{t} \partial_{j} u, \partial_{i} u\right) \\
& -\left(\left(\partial_{i} A_{i j}\right) \partial_{j} u+\left(\partial_{i} B_{i j}\right) \partial_{j} u_{t}, u_{t}+\delta u+\delta^{2} u_{t t}\right)+\delta^{2}\left(\left(A_{i j}\right)_{t} \partial_{j} u, \partial_{i} u_{t}\right) \\
& +\frac{\delta^{2}}{2}\left(\left(B_{i j}\right)_{t} \partial_{j} u_{t}, \partial_{i} u_{t}\right) .
\end{aligned}
$$

Then, by integration by parts, we have

$$
\frac{1}{2} \frac{d}{d t} E+F=\left(P(v) u, u_{t}+\delta u+\delta^{2} u_{t t}\right)+R .
$$

Using Poincaré's inequality and choosing $\delta>0$ small enough, we see that

$$
c_{1}\|U\|^{2} \leqq E \leqq c_{2}\|U\|^{2} \text { and } F \geqq c_{3}\left\{\|U\|^{2}+\left\|u_{t t}\right\|^{2}\right\}
$$

for suitable positive constants $c_{1}, c_{2}$ and $c_{3}$. And also, we have

$$
\begin{gathered}
|R| \leqq C p_{v}(t)\left\{\|U\|^{2}+\left\|u_{t t}\right\|^{2}\right\} \\
\left|\left(P(v) u, u_{t}+\delta u+\delta^{2} u_{t t}\right)\right| \leqq C\|P(v) u\|\left\{\|U\|+\left\|u_{t t}\right\|\right\} .
\end{gathered}
$$

Therefore, there exist $\beta_{1}$ and $\omega>0$ such that

$$
\frac{d}{d t} E+3 \omega\left(E+\left\|u_{t t}\right\|^{2}\right) \leqq C\|P(v) u\|^{2}
$$

provided that $p_{v}(t) \leqq \beta_{1}$ for $t \in[S, T]$. Since $e^{-2 \omega t} \frac{d}{d t}\left(e^{2 \omega t} E\right)=\frac{d}{d t} E+2 \omega E$, integration over $[S, t]$ and use of (3.2.3) imply the theorem immediately.

Corollary 3.2.2. Suppose that Assumption 1.1 is satisfied. Let $u$ and $v$ be two vectors of functions satisfying (3.2.1) and suppose that $P(v) u \in C^{1}\left([S, T] ; L^{2}\right)$. Then,

$$
\begin{gathered}
\Psi\left(\left\|U_{t}(t, \cdot)\right\|, \omega, S\right) \leqq C\left\{e^{2 \omega S}\left\|U_{t}(S, \cdot)\right\|^{2}+\int_{S}^{t} e^{2 \omega s}\left\|(P(v) u)_{t}(s, \cdot)\right\|^{2} d s\right. \\
+\int_{S}^{t} e^{2 \omega s} p_{v}(s)^{2}\left(\left\|\partial^{2} u(s, \cdot)\right\|^{2}+\left\|\partial_{x}^{2} \partial_{t} u(s, \cdot)\right\|^{2}\right) d s
\end{gathered}
$$

for $t \in[S, T]$ provided that $p_{v}(t) \leqq \beta_{1}$ for $t \in[S, T]$.

Proof. Using a mollifier with respect to $t$ only, we may assume that $u \in$ $C^{\infty}\left([S, T] ; H^{2}\right)$ and that $u=0$ on $[S, T] \times \partial \Omega$ (cf. Shibata [22, Lemma 4.1]). Differentiation of $P(v) u$ once in $t$ and application of Theorem 3.2.1 to the resulting equation imply the Corollary immediately. 
3.3. Estimate of Spatial Derivatives in Half Space. In this paragraph, as a preparation of the estimate of spatial derivatives in $\Omega$, we shall discuss the half space case. Let $u$ be a vector of function satisfying the following condition:

$$
Q u=g \text { in }[S, T] \times H_{+} \text {and } u=0 \text { on }[S, T] \times \partial H_{+} .
$$

Here, $\quad H_{+}=\left\{x \in \mathbb{R}^{n} \mid x_{n}>0\right\} . \quad \partial H_{+}=\left\{x \in \mathbb{R}^{n} \mid x_{n}=0\right\}, \quad Q u=a_{i j}(t, x) \partial_{i} \partial_{j} u+$ $b_{i j}(t, x) \partial_{i} \partial_{j} \partial_{t} u$ and the $a_{i j}$ and $b_{i j}$ are $d \times d$ matrices of real-valued functions satisfying the following assumption:

Assumption 3.3.1. (1) ${ }^{t} a_{i j}=a_{j i},{ }^{t} b_{i j}=b_{j i}$ and $a_{i j}, b_{i j} \in \bigcap_{j=0}^{1} C^{j}\left([S, T] ; H^{N-1-j}\right)$.

$$
\left(m_{i j} \partial_{j} u, \partial_{i} u\right) \geqq \beta_{3}\|\nabla u\|^{2} \quad \text { for any } u \in H_{0}^{1}
$$

for some constant $\beta_{3}>0$, where $m=a$ and $b$.

In this paragraph, in order to denote that usual $L^{2}$ space on $H_{+}$, its norm, its inner-product, the usual Sobolev space of order $j$ in the $L^{2}$ sense and its norm, we use the same notation: $L^{2},\|\cdot\|,(),, H^{j}$ and $\|\cdot\|_{j}$ as the notation defined in Sect. 2, respectively. And, we put

$$
H_{0}^{1}=\left\{u \in H^{1} \mid u=0 \text { on } \partial H_{+}\right\} \text {and }\|u\|_{\infty}=\sup _{x \in H_{+}}|u(x)| .
$$

Then, we have the following theorem.

Theorem 3.3.2. Suppose that Assumption 3.3 .1 is satisfied and that $\left\|\left(a_{i j}, b_{i j}\right)(t, \cdot)\right\|_{\infty} \leqq \beta_{4}$ for $t \in[S, T]$. Let $L$ be an integer $\in[2, N]$. Assume that $u$ satisfies (3.3.1) and that $u \in C^{1}\left([S, T] ; H^{L}\right)$. Then, there exist $\omega$ and $\beta_{5}$ depending only on $\beta_{3}$ and $\beta_{4}$ essentially such that

$$
\begin{gathered}
\Psi\left(\left\|\partial_{x}^{L}\left(u, u_{t}\right)(t, \cdot)\right\|, \omega, S\right) \leqq C\left(L, \beta_{3}, \beta_{4}\right)\left\{e^{2 \omega S}\left\|\partial_{x}^{L} u(S, \cdot)\right\|^{2}+\Psi\left(\|g(t, \cdot)\|_{L-2}, \omega, S\right)\right. \\
\left.+\Psi\left(\left\|\left(a_{i j}, b_{i j}\right)(t, \cdot)\right\|_{L(n)}\left\|\left(u, u_{t}\right)(t, \cdot)\right\|_{L-1}, \omega, S\right)\right\} \text { for any } t \in[S, T],
\end{gathered}
$$

provided that $\left\|\left(a_{i j}, b_{i j}\right)_{t}(t, \cdot)\right\|_{\infty} \leqq \beta_{5}$ for all $t \in[S, T]$, where $L(n)=\max ([n / 2]$ $+2, L-2)$.

Proof. Using the mollifier with respect to $\left(x_{1}, \ldots, x_{n-1}\right)$ and noting Friedrich's well-known lemma concerning the commutator of the mollifier and the multiplication of function, without loss of generality we may assume that $u$ is differentiable any times with respect to $\left(x_{1}, \ldots, x_{n-1}\right)$ in the course of proof below.

First, we shall prove the theorem for $L=2$. Let $l=1,2, \ldots, n-1$. Multiplying (3.3.1) by $\partial_{l}^{2}\left(u+u_{t}\right)$ and noting that $u=\partial_{l} u=\partial_{l}^{2} u=u_{t}=\partial_{l} u_{t}=\partial_{l}^{2} u_{t}=0$ on $\partial H_{+}$, by integration by parts we have

$$
\begin{aligned}
\left(g, \partial_{l}^{2}\left(u+u_{t}\right)\right)= & -\left(\left(\partial_{l} a_{i j}\right) \partial_{i} \partial_{j} u+\left(\partial_{l} b_{i j}\right) \partial_{i} \partial_{j} u_{t}, \partial_{l}\left(u+u_{t}\right)\right) \\
& +\left(\left(\partial_{i} a_{i j}\right) \partial_{l} \partial_{j} u+\left(\partial_{i} b_{i j}\right) \partial_{l} \partial_{j} u_{t}, \partial_{l}\left(u+u_{t}\right)\right) \\
& +\frac{1}{2} \frac{d}{d t}\left(\left(a_{i j}+b_{i j}\right) \partial_{j} \partial_{l} u, \partial_{i} \partial_{l} u\right) \\
& +\left(a_{i j} \partial_{j} \partial_{l} u, \partial_{i} \partial_{l} u\right)+\left(b_{i j} \partial_{j} \partial_{l} u_{t}, \partial_{i} \partial_{l} u_{t}\right) \\
& -\frac{1}{2}\left(\left(a_{i j}+b_{i j}\right)_{t} \partial_{j} \partial_{l} u, \partial_{i} \partial_{l} u\right)
\end{aligned}
$$


It follows from Schwarz's inequality and Assumption 3.3.1-(2) that

$$
\begin{gathered}
\frac{1}{2} \frac{d}{d t}\left(\left(a_{i j}+b_{i j}\right) \partial_{j} \partial_{l} u, \partial_{i} \partial_{l} u\right)+\beta_{3}\left(\left\|\nabla \partial_{l} u\right\|^{2}+\left\|\nabla \partial_{l} u_{t}\right\|^{2}\right) \\
\leqq C\left\{\|g\|\left\|\partial_{l}^{2}\left(u+u_{t}\right)\right\|+\left\|\left(a_{i j}, b_{i j}\right)_{t}\right\|_{\infty}\left\|\nabla \partial_{l} u\right\|^{2}\right. \\
\left.\quad+\left\|\partial_{x}^{1}\left(a_{i j}, b_{i j}\right)\right\|_{\infty}\left(\left\|\partial_{x}^{2} u\right\|+\left\|\partial_{x}^{2} u_{t}\right\|\right)\left\|\nabla\left(u+u_{t}\right)\right\|\right\} .
\end{gathered}
$$

For $2^{\text {nd }}$ order normal derivatives, we have

$$
\begin{aligned}
\left(a_{n n} \partial_{n}^{2} u+b_{n n} \partial_{n}^{2} u_{t}, \partial_{n}^{2}\left(u+u_{t}\right)\right)= & \left(a_{n n} \partial_{n}^{2} u, \partial_{n}^{2} u\right)+\left(b_{n n} \partial_{n}^{2} u_{t}, \partial_{n}^{2} u_{t}\right) \\
& +\frac{1}{2} \frac{d}{d t}\left(\left(a_{n n}+b_{n n}\right) \partial_{n}^{2} u, \partial_{n}^{2} u\right) \\
& -\frac{1}{2}\left(\left(a_{n n}+b_{n n}\right)_{t} \partial_{n}^{2} u, \partial_{n}^{2} u\right) .
\end{aligned}
$$

It is known that $a_{n n} \geqq \beta_{3} I_{d}$ and $b_{n n} \geqq \beta_{3} I_{d}$, which follows from Assumption 3.3.1-(2) (cf. Shibata [22, Lemma 3.4]). Let $\delta$ be a small positive number determined later. Combining (3.3.2) and (3.3.3) implies that

$$
\begin{aligned}
\frac{1}{2} \frac{d}{d t}\left\{\sum _ { l = 1 } ^ { n - 1 } \left(\left(a_{i j}\right.\right.\right. & \left.\left.\left.+b_{i j}\right) \partial_{j} \partial_{l} u, \partial_{i} \partial_{l} u\right)+\delta\left(\left(a_{n n}+b_{n n}\right) \partial_{n}^{2} u, \partial_{n}^{2} u\right)\right\} \\
& +\beta_{3}\left\{\sum_{l=1}^{n-1}\left\|\nabla \partial_{l}\left(u, u_{t}\right)\right\|^{2}+\delta\left\|\partial_{n}^{2}\left(u, u_{t}\right)\right\|^{2}\right\} \\
\leqq & C\left\{\delta \sum_{l=1}^{n-1}\left\|\nabla \partial_{l}\left(u, u_{t}\right)\right\|\left\|\partial_{n}^{2}\left(u, u_{t}\right)\right\|+\|g\|\left\|\partial_{x}^{2}\left(u, u_{t}\right)\right\|\right. \\
& \left.+\left\|\left(a_{i j}, b_{i j}\right)_{t}\right\|\left\|\partial_{x}^{2}\left(u, u_{t}\right)\right\|^{2}+\left\|\partial_{x}^{1}\left(a_{i j}, b_{i j}\right)\right\|_{\infty}\left\|\partial_{x}^{2}\left(u, u_{t}\right)\right\|\left\|\nabla\left(u, u_{t}\right)\right\|\right\} .
\end{aligned}
$$

Choosing $\delta>0$ small enough, we see easily that there exist $\omega$ and $\beta_{5}$ depending only on $\beta_{3}$ and $\beta_{4}$ essentially such that

$$
\begin{aligned}
& \Psi\left(\left\|\partial_{x}^{2} u(t, \cdot)\right\|, \omega, S\right)+\int_{S}^{t} e^{2 \omega s}\left\|\partial_{x}^{2} u_{t}(s, \cdot)\right\|^{2} d s \\
& \leqq \\
& \quad+\left\{e^{2 \omega S}\left\|\partial_{x}^{2} u(S, \cdot)\right\|^{2}+\int_{S}^{t} e^{2 \omega s}\|g(s, \cdot)\|^{2} d s\right. \\
& \quad+\int_{S}^{t} e^{2 \omega s}\left\|\partial_{x}^{1}\left(a_{i j}, b_{i j}\right)(s, \cdot)\right\|_{\infty}^{2}\left\|\nabla\left(u, u_{t}\right)(s, \cdot)\right\|^{2} d s,
\end{aligned}
$$

provided that $\left\|\left(a_{i j}, b_{i j}\right)_{t}(s, \cdot)\right\|_{\infty} \leqq \beta_{5}$ for any $s \in[S, T]$. Considering $\left(b_{i j} \partial_{i} \partial_{j} u_{t}, \partial_{l}^{2} u_{t}\right)(l=1, \ldots, n-1)$ and $\left(b_{n n} \partial_{n}^{2} u_{t}, \partial_{n}^{2} u_{t}\right)$ and employing the similar arguments, we have also

$$
\left\|\partial_{x}^{2} u_{t}\right\|^{2} \leqq C\left(\left\|\partial_{x}^{2} u\right\|^{2}+\|g\|^{2}+\left\|\partial_{x}^{1} b_{i j}\right\|_{\infty}^{2}\left\|\nabla u_{t}\right\|^{2}\right)
$$

Combining (3.3.4) and (3.3.5) and using Sobolev's imbedding theorem implies that the theorem is valid for $L=2$. 
Now, we shall evaluate higher order derivatives. Assume that $\left\|\left(a_{i j}, b_{i j}\right)_{t}(t, \cdot)\right\|_{\infty}$ $\leqq \beta_{5}$ for any $t \in[S, T]$, below. For any multi-index $\alpha=\left(\alpha_{1}, \ldots, \alpha_{n}\right)$ with $|\alpha|$ $=L-2$, put $h_{\alpha}=\partial_{x}^{\alpha} g-\left[\partial_{x}^{\alpha}, Q\right] u$. Applying Lemma 3.1.2-(2) to $h_{\alpha}$ implies that

$$
\left\|h_{\alpha}\right\| \leqq\left\|\partial_{x}^{\alpha} g\right\|+C(L)\left\|\left(a_{i j}, b_{i j}\right)\right\|_{L(n)}\left\|\left(u, u_{t}\right)\right\|_{L-1} .
$$

With the help of (3.3.6), differentiation of (3.3.1) with respect to $\left(x_{1}, \ldots, x_{n-1}\right)$ and application of the theorem for $L=2$ to the resulting formula yields:

$$
\begin{aligned}
& \Psi\left(\left\|\partial_{x}^{2} \partial_{x}^{\alpha^{\prime}}\left(u, u_{t}\right)(t, \cdot)\right\|, \omega, S\right) \\
& \leqq C\left\{e^{2 \omega S}\left\|\partial_{x}^{2} \partial_{x}^{\alpha^{\prime}} u(S, \cdot)\right\|^{2}+\Psi\left(\left\|\partial_{x}^{L-2} g(t, \cdot)\right\|, \omega, S\right)\right. \\
& \left.+\Psi\left(\left\|\left(a_{i j}, b_{i j}\right)(t, \cdot)\right\|_{L(n)}\left\|\left(u, u_{t}\right)(t, \cdot)\right\|_{L-1}, \omega, S\right)\right\}
\end{aligned}
$$

for any $\alpha^{\prime}=\left(\alpha_{1}, \ldots, \alpha_{n-1}, 0\right)$ with $\left|\alpha^{\prime}\right|=L-2$. Integrating the inner-product: $\left(a_{n n} \partial_{n}^{2} \partial_{x}^{\alpha} u+b_{n n} \partial_{n}^{2} \partial_{x}^{\alpha} u_{t}, \partial_{n}^{2} \partial_{x}^{\alpha}\left(u+u_{t}\right)\right)$ with respect to $t$ and adding the innerproduct: $\left(b_{n n} \partial_{n}^{2} \partial_{x}^{\alpha} u_{t}, \partial_{n}^{2} \partial_{x}^{\alpha} u_{t}\right)$, with the help of (3.3.6) we have

$$
\begin{aligned}
\Psi\left(\left\|\partial_{n}^{2} \partial_{x}^{\alpha}\left(u, u_{t}\right)(t, \cdot)\right\|, \omega, S\right) \leqq & C\left\{e^{2 \omega S}\left\|\partial_{n}^{2} \partial_{x}^{\alpha} u(S, \cdot)\right\|^{2}+\Psi\left(\left\|\partial_{x}^{L-2} g(t, \cdot)\right\|, \omega, S\right)\right. \\
& +\Psi\left(\left\|\left(a_{i j}, b_{i j}\right)(t, \cdot)\right\|_{L(n)}\left\|\left(u, u_{t}\right)(t, \cdot)\right\|_{L-1}, \omega, S\right) \\
& +\sum_{j=1}^{n-1} \Psi\left(\left\|\partial_{j} \partial_{n} \partial_{x}^{\alpha}\left(u, u_{t}\right)(t, \cdot)\right\|, \omega, S\right) \\
& \left.+\sum_{i, j=1}^{n-1} \Psi\left(\left\|\partial_{i} \partial_{j} \partial_{x}^{\alpha}\left(u, u_{t}\right)(t, \cdot)\right\|, \omega, S\right)\right\}
\end{aligned}
$$

for $\alpha=\left(\alpha_{1}, \ldots, \alpha_{n}\right)$ with $|\alpha|=L-2$. Repeated use of (3.3.8) and combination of the resulting inequality with (3.3.7) in the final step implies the theorem.

3.4. Estimate of Spatial Derivatives in Case of $\Omega$. Here, we shall discuss the estimate of spatial derivatives in $[S, T] \times \Omega$. Let $D$ be an open set such that $D \cap \Omega$ is non-empty and $\Phi$ a $C^{\infty}$-diffeomorphism from $D$ to $D^{\prime}=\Phi(D)$. Let $\Phi \in C_{0}^{\infty}(D)$ and put $(\phi u)(t, x)=w(t, y)$, where $y=\Phi(x)$. Since

$$
\begin{aligned}
A_{i j}(V) \partial_{i} \partial_{j}(\phi u)+B_{i j} \partial_{i} \partial_{j}\left(\phi u_{t}\right)= & A_{i j}(V)\left\{\left(\partial_{i} \phi\right) \partial_{j} u+\left(\partial_{j} \phi\right) \partial_{i} u+\left(\partial_{i} \partial_{j} \phi\right) u\right\} \\
& +B_{i j}(V)\left\{\left(\partial_{i} \phi\right) \partial_{j} u_{t}+\left(\partial_{j} \phi\right) \partial_{i} u_{t}+\left(\partial_{i} \partial_{j} \phi\right) u_{t}\right\} \\
& -\phi\left(P(v) u-A_{0}(V) \partial_{t}^{2} u-A_{j}(V) \partial_{j} \partial_{t} u\right)
\end{aligned}
$$

we have

$$
a_{k l} \partial_{k} \partial_{l} w+b_{k l} \partial_{k} \partial_{l} \partial_{t} w=g_{\phi} \circ \Phi^{-1}
$$

where

$$
\begin{aligned}
m_{k l}=M_{i j}(V \circ & \left.\Phi^{-1}\right)\left(\partial y_{k} / \partial x_{i}\right)\left(\partial y_{l} / \partial x_{j}\right)(m=a \text { and } b, M=A \text { and } B, \text { respectively }) \\
g_{\phi}= & -\phi\left(P(v) u-A_{0}(V) \partial_{t}^{2} u-A_{j}(V) \partial_{j} \partial_{t} u\right)+A_{i j}(V)\left(\left(\partial_{i} \phi\right) \partial_{j} u\right. \\
& \left.+\left(\partial_{j} \phi\right) \partial_{i} u+\left(\partial_{i} \partial_{j} \phi\right) u\right)+B_{i j}(V)\left(\left(\partial_{i} \phi\right) \partial_{j} u_{t}+\left(\partial_{j} \phi\right) \partial_{i} u_{t}\right. \\
& \left.+\left(\partial_{i} \partial_{j} \phi\right) u_{t}\right)-A_{i j}(V)\left(\partial^{2} y_{l} / \partial x_{i} \partial x_{j}\right)\left(\partial w / \partial y_{l}\right) \\
& -B_{i j}(V)\left(\partial^{2} y_{l} / \partial x_{i} \partial x_{j}\right)\left(\partial w_{t} / \partial y_{l}\right)
\end{aligned}
$$


Applying Lemma 3.1.2-(2) to $g_{\phi}$ implies that

$$
\begin{aligned}
\left\|g_{\phi}\right\|_{L-2} \leqq & C(\phi, \Phi, L)\left\{\|P(v) u\|_{L-2}+\left\|A_{0}(V)\right\|_{\infty}\left\|\partial_{x}^{L-2} \partial_{t}^{2} u\right\|\right. \\
& \left.+\left\|\left(A_{j}(V), A_{i j}(V), B_{i j}(V)\right)\right\|_{L(n)}\left(\left\|\partial_{t}^{2} u\right\|_{L-3}+\left\|\left(u, u_{t}\right)\right\|_{L-1}\right)\right\} .
\end{aligned}
$$

It follows from (2.4) that

$$
\int_{D^{\prime}} m_{k l} \partial_{k} w \partial_{l} w d y \geqq \delta_{\phi} \int_{D^{\prime}}|\nabla w|^{2} d y
$$

with some constant $\delta_{\phi}=C\left(\phi, \Phi, \alpha_{0}\right)>0$ for any $w(y)=u\left(\Phi^{-1}(y)\right)$ with $u \in H_{0}^{1}$. Therefore, using a partition of unity of $\bar{\Omega}$ consisting of a finite number of functions in $C_{0}^{\infty}\left(\mathbb{R}^{n}\right)$ and applying Theorem 3.3.2, we have the following theorem.

Theorem 3.4.1. Suppose that Assumption 1.1 is satisfied. Let $u$ and $v$ be two vectors of functions satisfying the following:

$$
v \in E_{N}([S, T]), \quad u \in E_{N}([S, T]), \quad\|V(t, \cdot)\|_{\infty} \leqq K \quad \text { for } t \in[S, T],
$$

where $L$ is an integer $\in[2, N]$. Then, there exist constants $\omega$ and $\beta_{6}>0$ such that

$$
\begin{aligned}
& \Psi\left(\left\|\partial_{x}^{L}\left(u, u_{t}\right)(t, \cdot)\right\|, \omega, S\right) \\
\leqq & C(L, \Omega)\left\{e^{2 \omega S}\|u(S, \cdot)\|_{L}^{2}+\Psi\left(\|P(v) u(t, \cdot)\|_{L-2}, \omega, S\right)+\Psi\left(\left\|\partial_{x}^{L-2} u_{t t}(t, \cdot)\right\|, \omega, S\right)\right. \\
& +\Psi\left(( 1 + \| ( A _ { j } ( V ) , A _ { i j } ( V ) , B _ { i j } ( V ) ) ( t , \cdot ) \| _ { L ( n ) } ) \left(\left\|\partial_{t}^{2} u(t, \cdot)\right\|_{L-3}\right.\right. \\
& \left.\left.\left.+\left\|\left(u, u_{t}\right)(t, \cdot)\right\|_{L-1}\right), \omega, S\right)\right\},
\end{aligned}
$$

provided that $p_{v}(t) \leqq \beta_{6}$ for any $t \in[S, T]$.

Remark 3.4.2. Since $\left\|\partial_{x}^{L-1} U(t, \cdot)\right\| \leqq\left\|\partial_{x}^{L-1}\left(u, u_{t}\right)(t, \cdot)\right\|+\left\|\partial_{x}^{L}\left(u, u_{t}\right)(t, \cdot)\right\|$, $\left\|\partial_{x}^{L-2} u_{t t}(t, \cdot)\right\| \leqq\left\|\partial_{x}^{L-2} U_{t}(t, \cdot)\right\|,\left\|\partial_{t}^{2} u(t, \cdot)\right\|_{L-3}+\left\|\left(u, u_{t}\right)(t, \cdot)\right\|_{L-1} \leqq C\left\|\partial^{L-2} U(t, \cdot)\right\|$ provided $u=0$ on $\partial \Omega$, the estimate in Theorem 3.4.1 can be rewritten as follows:

$$
\begin{aligned}
& \Psi\left(\left\|\partial_{x}^{L-1} U(t, \cdot)\right\|, \omega, S\right) \\
\leqq & C(L, \Omega)\left\{e^{2 \omega S}\|u(S, \cdot)\|_{L}^{2}+\Psi\left(\|P(v) u(t, \cdot)\|_{L-2}, \omega, S\right)+\Psi\left(\left\|\partial_{x}^{L-2} U_{t}(t, \cdot)\right\|, \omega, S\right)\right. \\
& \left.+\Psi\left(\left(1+\left\|\left(A_{j}(V), A_{i j}(V), B_{i j}(V)\right)(t, \cdot)\right\|_{L(n)}\right)\left\|\bar{\partial}^{L-2} U(t, \cdot)\right\|, \omega, S\right)\right\}
\end{aligned}
$$

for $2 \leqq L \leqq N$.

\section{A Priori Estimate}

In this section, we shall discuss the a priori estimate of $u$ satisfying the following:

$$
\begin{gathered}
P(v) u=f \quad \text { in }[S, T] \times \Omega \text { and } u=0 \text { on }[S, T] \times \partial \Omega, \\
v \in E_{N}([S, T]), \quad u \in E_{L}([S, T])(2 \leqq L \leqq N), \\
f \in C^{L-1}\left([S, T] ; L^{2}\right) \cap \bigcap_{j=0}^{L-2} C^{j}\left([S, T] ; H^{L-2-j}\right), \\
\|V(t, \cdot)\|_{\infty} \leqq K \text { and } p_{v}(t) \leqq 1 \text { for } t \in[S, T]
\end{gathered}
$$


We shall prove the following theorem.

Theorem 4.1. Suppose that Assumption 1.1 is satisfied. Let $u, v$ and $f$ be three vectors of functions satisfying (4.1), (4.2) and (4.3). Then, there exist constants $\omega$ and $\beta>0$ such that

$$
\begin{aligned}
\Psi\left(\left\|\bar{\partial}^{k-1} U(t, \cdot)\right\|, \omega, S\right) \leqq & C(L)\left\{e^{2 \omega S}\left(\left\|\bar{\partial}^{k} u(S, \cdot)\right\|^{2}+\left\|\nabla \partial_{t}^{k} u(S, \cdot)\right\|^{2}\right)\right. \\
& +\Psi\left(\left\|\bar{\partial}^{k-1} f(t, \cdot)\right\|, \omega, S\right)+\int_{S}^{t} e^{2 \omega s}\left\|\partial_{t}^{k-1} f(s, \cdot)\right\|^{2} d s \\
& +\Psi\left(Q_{k}(v)(t)\left\|\bar{\partial}^{k-2} U(t, \cdot)\right\|, \omega, S\right) \\
& \left.+\int_{S}^{t} e^{2 \omega s} R_{k}(u, v)(s)^{2} d s\right\},
\end{aligned}
$$

provided that $p_{v}(t) \leqq \beta$ for $t \in[S, T]$, where

$$
\begin{aligned}
& Q_{k}(v)(t)= \begin{cases}1 & \text { for } k \leqq N_{0}, \\
1+\left(1+\left\|\bar{\partial}^{k-2} V(t, \cdot)\right\|\right)^{k-3}\left\|\bar{\partial}^{k-2} V(t, \cdot)\right\| & \text { for } k \geqq N_{0}+1,\end{cases} \\
& R_{k}(u, v)(t) \\
& = \begin{cases}p_{v}(t)\left\|\bar{\partial}^{k-1} U(t, \cdot)\right\| & \text { for } k \leqq N_{0}, \\
p_{v}(t)\left\|\bar{\partial}^{k-1} U(t, \cdot)\right\|+p_{u}(t)\left\|\bar{\partial}^{k-1} V(t, \cdot)\right\| & \text { for } k \geqq N_{0}+1 . \\
+\left(1+\left\|\bar{\partial}^{k-2} V(t, \cdot)\right\|\right)^{k-2}\left\|\bar{\partial}^{k-2} V(t, \cdot)\right\|\left\|\bar{\partial}^{k-2} U(t, \cdot)\right\|\end{cases}
\end{aligned}
$$

Proof. Let $k$ be an integer $\in[2, L]$. Differentiating (4.1) $k-2$ times with respect to $t$, we can write the resulting equation as follows:

$$
P(v) \partial_{t}^{k-2} u=\partial_{t}^{k-2}-F_{k} \text { in }[S, T] \times \Omega, \partial_{t}^{k-2} u=0 \text { on }[S, T] \times \partial \Omega,
$$

where $F_{k}=\left[\partial_{t}^{k-2}, P(v)-P(0)\right] u$. Applying Lemmas 3.1.1 and 3.1.2-(1) to $F_{k}$ and using (4.3) and the fact that

$$
\left\|\bar{\partial}^{l}\left(u_{t t}, \nabla u_{t}, \partial_{x}^{2} u, \partial_{x}^{2} u_{t}\right)(t, \cdot)\right\| \leqq C(l)\left\|\bar{\partial}^{l+1} U(t, \cdot)\right\|
$$

we have

$$
\left\|\bar{\partial}^{1} F_{k}(t, \cdot)\right\| \leqq C(k) R_{k}(u, v)(t) .
$$

Applying Corollary 3.2.2 to (4.4) implies that

$$
\begin{gathered}
\Psi\left(\left\|\partial_{t}^{k-1} U(t, \cdot)\right\|, \omega, S\right) \leqq C(k)\left\{e^{2 \omega S}\left\|\partial_{t}^{k-1} U(S, \cdot)\right\|^{2}\right. \\
\left.+\int_{S}^{t} e^{2 \omega s}\left(\left\|\partial_{t}^{k-1} f(s, \cdot)\right\|^{2}+R_{k}(u, v)(s)^{2}\right) d s\right\},
\end{gathered}
$$

provided that $p_{v}(t) \leqq \beta_{1}$ for $t \in[S, T]$. In order to evaluate $\left\|\partial_{x}^{k-1-l} \partial_{t}^{l} U(t, \cdot)\right\|$ $(0 \leqq l \leqq k-2)$, let us differentiate (4.1) $l$ times with respect to $t$ and apply (3.4.4) to 
the resulting equation with $L=k-l$. For estimate of the nonlinear term, we apply Lemma 3.1.1 and use (4.3), and then we have

$$
\begin{gathered}
\left\|\left(A_{j}(V), A_{i j}(V), B_{i j}(V)\right)(t, \cdot)\right\|_{k(n)} \leqq C(k) Q_{k}(v)(t), \\
\left\|\partial_{k}^{l}((P(v)-P(0)) u)-(P(v)-P(0)) \partial_{t}^{l} u\right\|_{k-l-2} \leqq C(k) Q_{k}(v)(t)\left\|\bar{\partial}^{k-2} U(t, \cdot)\right\|,
\end{gathered}
$$

where $k(n)=\max ([n / 2]+2, k-2)$.

Therefore, it follows that

$$
\begin{aligned}
\Psi\left(\left\|\partial_{x}^{k-1-l} \partial_{t}^{l} U(t, \cdot)\right\|, \omega, S\right) \leqq & C(k)\left\{e^{2 \omega S}\left\|\partial_{x}^{k-l} \partial_{t}^{l} u(S, \cdot)\right\|^{2}\right. \\
& +\Psi\left(\left\|\partial_{t}^{l} f(t, \cdot)\right\|_{k-2-l}, \omega, S\right) \\
& +\Psi\left(\left\|\partial_{x}^{k-2-l} \partial_{t}^{l+1} U(t, \cdot)\right\|, \omega, S\right) \\
& \left.+\Psi\left(Q_{k}(v)(t)\left\|\bar{\partial}^{k-2} U(t, \cdot)\right\|, \omega, S\right)\right\},
\end{aligned}
$$

provided that $p_{v}(t) \leqq \beta_{6}$ for $t \in[S, T]$. Repeated use of (4.7), and substitution of (4.6) and use of Theorem 3.2.1 in the final step imply the theorem immediately.

\section{A Proof of Theorem 2.2}

Our proof of the existence part (A) of Theorem 2.2 consists in combining the following local existence and uniqueness theorem with uniform a priori estimate.

Theorem 5.1 (local existence and uniqueness). Suppose that Assumptions 1.1 and 2.1 are satisfied. Then, there exists a T>0 depending only on $\rho_{N_{0}}+\lambda_{N_{0}}(\infty)$ essentially such that the initial boundary value problem:

$$
\begin{gathered}
p(u) u=f \text { in }[0, T] \times \Omega \text { and } u=0 \text { on }[0, T] \times \partial \Omega, \\
u(0, x)=u_{0}(x) \text { and } u_{t}(0, x)=u_{1}(x) \text { in } \Omega,
\end{gathered}
$$

admits a unique solution $u \in E_{N}([0, T])$ satisfying the condition: $\|U(t, \cdot)\|_{\infty} \leqq 2 K / 3$ for $t \in[0, T]$ provided that $\left\|\left(\nabla u_{0}, u_{1}, \nabla u_{1}\right)\right\|_{\infty} \leqq K / 2$.

Since we have already shown how to get the a priori estimate for $P(v) u$, by using the usual contraction method we can prove Theorem 5.1 in a standard way (cf. Shibata-Kikuchi [23] or Kato [16]). We will write a proof of Theorem 5.1 elsewhere.

Now, let us prove a global existence of the solution to the problem $(1.1-(1.3)$. Let $\varepsilon>0$ be a small constant determined later and assume that $u \in E_{N}([0, T])$ and that $u$ satisfies (5.1) and the following:

$$
p_{u}(t) \leqq \varepsilon \quad \text { for } 0 \leqq t \leqq T .
$$

Assume that $\varepsilon \leqq \beta$ (cf. Theorem 4.1), that $\varepsilon \leqq \beta_{1}$ (cf. Theorem 3.2.1) and that $\alpha_{2} \varepsilon \leqq K / 2$ (cf. (2.2)). In particular, we have by (2.2)

$$
\left\|\bar{\partial}^{1} U(t, \cdot)\right\|_{\infty} \leqq \alpha_{2} p_{u}(t) \leqq \alpha_{2} \varepsilon \leqq K / 2 .
$$


With the help of Theorem 3.2.1, repeated use of Theorem 4.1 implies that there exists a $\omega>0$ such that

$$
\begin{gathered}
e^{2 \omega t} p_{u}(t)^{2}+\int_{0}^{t} e^{2 \omega s} p_{u}(s)^{2} d s \leqq C(n)\left\{\rho_{N_{0}}^{2}+C(\gamma) e^{2(\omega-\gamma) t} \lambda_{N_{0}}(\infty)^{2}\right. \\
\left.+\varepsilon^{2} \int_{0}^{t} e^{2 \omega s} p_{u}(s)^{2} d s\right\} \quad \text { for } 0 \leqq t \leqq T .
\end{gathered}
$$

Therefore, if $\varepsilon$ is chosen additionally in such a way that $C(n) \varepsilon^{2} \leqq 1$, we have

$$
\left\|\bar{\partial}^{[n / 2]+2} U(t, \cdot)\right\| \leqq C(n)\left\{e^{-\omega t} \rho_{N_{0}}+C(\gamma) e^{-\varepsilon t} \lambda_{N_{0}}(\infty)\right\} .
$$

If $C(n)\left\{\rho_{N_{0}}+C(\gamma) \lambda_{N_{0}}(\infty)\right\} \leqq \varepsilon / 2$, then $p_{u}(t) \leqq \varepsilon / 2$ for $0 \leqq t \leqq T$. Therefore, by Theorem 5.1 we can continue $u \in E_{N}([0, T])$ to a $u^{\prime} \in E_{N}\left(\left[0, T^{\prime}\right]\right)$ with some $T^{\prime}>T$ in a unique way such that $u^{\prime}$ satisfies (5.1) and (5.2), replacing $T$ by $T^{\prime}$ there. In addition, since $p_{u}(0) \leqq C \tilde{\rho}_{N_{0}}$ for a suitable constant $C>0$ provided that $u \in$ $E_{N}([0, T])$ and $u$ satisfies $(5.1)$, if $\tilde{\rho}_{N_{0}} \leqq \varepsilon / 2 C$, then Theorem 5.1 and the continuity of $p_{u}(t)$ imply that there exists a $T>0$ such that (5.1) admits a unique solution $u \in E_{N}([0, T])$ which satisfies (5.2). Combining these facts, we can continue a solution locally in time to any time interval, which completes the proof of the part (A) of Theorem 2.2 .

Now, we shall show part (B) of Theorem 2.2. From (5.5) it follows that (2.8) and (2.9) are valid for $k=N_{0}$. Assume that $k \geqq N_{0}+1$ and that (2.8) and (2.9) are valid for smaller values of $k$. Since $p_{u}(t) \leqq \varepsilon$ for any $t \in[0, \infty)$, applying Theorem 4.1 and using (5.5), we have

$$
\begin{aligned}
& e^{2 \omega t}\left\|\bar{\partial}^{k-1} U(t, \cdot)\right\|^{2}+\int_{0}^{t} e^{2 \omega s}\left\|\bar{\partial}^{k-1} U(s, \cdot)\right\|^{2} d s \\
& \leqq \\
& \quad C(k)\left\{\rho_{k}^{2}+C(\gamma) e^{2(\omega-\gamma) t} \lambda_{k}(\infty)^{2}\right. \\
& \left.\quad+\Psi\left(\left(1+\left\|\bar{\partial}^{k-2} U(t, \cdot)\right\|\right)^{k-2}\left\|\bar{\partial}^{k-2} U(t, \cdot)\right\|, \omega, 0\right)\right\} \\
& \quad+C(k) \rho_{N_{0}}^{2} \int_{0}^{t} e^{-2 \omega s}\left(\left\|\bar{\partial}^{k-1} U(s, \cdot)\right\|^{2} e^{2 \omega s}\right) d s \\
& \quad+C(k, \gamma) \lambda_{N_{0}}(\infty)^{2} \int_{0}^{t} e^{-2 \omega s}\left(\left\|\bar{\partial}^{k-1} U(s, \cdot)\right\|^{2} e^{2 \omega s}\right) d s
\end{aligned}
$$

When $\gamma=0$ (i.e., $\mu=0$ ), we choose $\lambda_{N_{0}}(\infty)$ in such a way that $C(k, 0) \lambda_{N_{0}}(\infty)^{2} \leqq 1$. Using Gronwall's inequality and the induction assumption and noting that we may assume that $\rho_{N_{0}}, \lambda_{N_{0}}(\infty) \leqq 1$, we have (2.8) and (2.9) for required $k$, which completes the proof of Theorem 2.2.

\section{A Proof of Theorem 2.3}

In this section, we shall prove an existence of periodic solution by using a standard method (see, Rabinowitz [21] and Matsumura [17]). For the readers' convenience, we shall give a proof of Theorem 2.3 , below. 
In order to construct a periodic solution, we consider a sequence $\left\{u^{m}\right\}$, where the $u^{m}$ satisfy the following:

$$
\begin{gathered}
u^{m} \in E_{N}(\mathbb{R}),\left\|U^{n}(t, \cdot)\right\|_{\infty} \leqq K / 2 \text { for } t \in \mathbb{R}\left(U^{m}=\left(\nabla u^{m}, u_{t}^{m}, \nabla u_{t}^{m}\right)\right), \\
P\left(u^{m}\right) u^{m}=f^{m} \text { in } \mathbb{R} \times \Omega \text { and } u^{m}=0 \text { on } \mathbb{R} \times \partial \Omega, \\
\left\|\bar{\partial}^{k-1} U^{m}(t, \cdot)\right\| \leqq C(k) \lambda_{k}(\infty) \text { for } t \in \mathbb{R} \text { and } N_{0} \leqq k \leqq N,
\end{gathered}
$$

where $f^{m}(t, x)=\rho(t+m) f(t, x)$ and $\rho(t) \in C^{\infty}(\mathbb{R})$ such that $\rho(t)=1$ for $t \geqq 1$ and $=0$ for $t \leqq 1 / 2$. The $u^{m}$ are defined by the following:

$$
u^{m}(t, x)=v^{m}(t+m, x) \text { for } t \geqq-m \text { and }=0 \text { for } t \leqq-m,
$$

where the $v^{m}$ are vectors of functions satisfying the following:

$$
\begin{gathered}
v^{m} \in E_{N}([0, \infty)), \quad\left\|V^{m}(t, \cdot)\right\|_{\infty} \leqq K / 2 \quad \text { for } t \in[0, \infty) \\
\left(V^{m}=\left(\nabla v^{m}, v_{t}^{m}, \nabla v_{t}^{m}\right)\right), \\
P\left(v^{m}\right) v^{m}=f^{m}(t-m, \cdot) \quad \text { in }[0, \infty) \times \Omega \text { and } v^{m}=0 \text { on }[0, \infty) \times \partial \Omega, \\
v^{m}(0, x)=v_{t}^{m}(0, x)=0 \text { in } \Omega, \\
\left\|\bar{\partial}^{k-1} V^{m}(t, \cdot)\right\| \leqq C(k) \lambda_{k}(\infty) \text { for } t \in[0, \infty) \text { and } N_{0} \leqq k \leqq N .
\end{gathered}
$$

Since $\operatorname{supp} f^{m}(t-m, \cdot) \subset\left[\frac{1}{2}, \infty\right) \times \bar{\Omega}$, the compatibility condition is satisfied for (6.5). And then, the existence of the $v^{m}$ follows from Theorem 2.2 under the assumption that $\lambda_{N_{0}}(\infty)$ is very small. Since $f$ is assumed to be bounded with respect to $t$ only, the way of choosing $\lambda_{N_{0}}(\infty)$ depends on $N$ in order to get (6.6) (cf. Theorem $2.2(\mathrm{~B})-(1))$. Since $\partial_{t}^{l} v^{m}(0, x)=0$ for $0 \leqq l \leqq N,(6.1),(6.2)$ and (6.3) follows from (6.4), (6.5) and (6.6), respectively.

Now, we shall show that $\left\{u^{m}(t, \cdot)\right\}$ is a Cauchy sequence in $E_{N-1}(\mathbb{R})$. Put

$$
w^{m}=u^{m+p}-u^{m} \quad \text { for any } m \text { and } p>0 \text { and } W^{m}=\left(\nabla w^{m}, w_{t}^{m}, \nabla w_{t}^{m}\right) .
$$

Then, the $w^{m}$ satisfy the equation:

$$
P\left(u^{m}\right) w^{m}=f^{m+p}-f^{m}+\left(P\left(u^{m}\right)-P\left(u^{m+p}\right)\right) u^{m+p} \quad \text { in } \mathbb{R} \times \Omega .
$$

Using Lemmas 3.1.2-(2) and 3.1.1, the well-known inequality:

$$
\|f \cdot g\|_{l} \leqq C(l)\|f\|_{l(n)}\|g\|_{l} \quad(l(n)=\max ([n / 2]+1, l)),
$$

and (6.3), we have

$$
\begin{aligned}
& \left\|\bar{\partial}^{k-2}\left(P\left(u^{m}\right)-P\left(u^{m+p}\right)\right) u^{m+p}\right\| \\
& \quad \leqq C\left(k, \lambda_{N}(\infty)\right)\left\|\bar{\partial}^{k-2} W^{m}(t, \cdot)\right\| \quad \text { for } 2 \leqq k \leqq N, \\
& \quad \leqq\left\{\begin{array}{l}
C(k) \lambda_{N_{0}}(\infty)\left\|\partial_{t}^{k-1} W^{m}(t, \cdot)\right\| \\
+C\left(k, \lambda_{N}(\infty)\right)\left\|\bar{\partial}^{k-2} W^{m}(t, \cdot)\right\| \text { for } 2 \leqq k \leqq N-1, \\
C(k) \lambda_{N_{0}}(\infty)\left\|W^{m}(t, \cdot)\right\| \text { for } k=1,
\end{array}\right.
\end{aligned}
$$

In the same manner as in Sect. 5, applying Theorems 4.1 and 3.2.1, noting that $\left(f^{m+p}-f^{m}\right)(t, \cdot)=0$ for $t \geqq-m+1$ and using (6.8) and (6.9), we see that

$$
\Psi\left(\left\|\bar{\partial}^{N-1} W^{m}(t, \cdot)\right\|, \omega, S\right) \leqq C\left(N, \lambda_{N}(\infty)\right) e^{2 \omega S}\left\|\bar{\partial}^{N-2} W^{m}(S, \cdot)\right\|^{2}
$$


for $t \geqq S \geqq-m+1$ with suitable $\omega>0$ provided that $\lambda_{N_{0}}(\infty)$ is small enough. For any $t \in \mathbb{R}$ and $m$ with $T \geqq-m+1$, it follows from (6.10) that

$$
\left\|\bar{\partial}^{N-2} W^{m}(T, \cdot)\right\| \leqq C\left(N, \lambda_{N}(\infty)\right) e^{-\omega(T+m-1)} \lambda_{N}(\infty),
$$

because $\left\|\bar{\partial}^{N-2} W^{m}(-m+1, \cdot)\right\| \leqq C(N) \lambda_{N}(\infty)$, which follows from (6.3). Using (6.10) for any $t>T$ again, we have

$$
\sup _{t \geqq T}\left\|\bar{\partial}^{N-2} W^{m}(t, \cdot)\right\| \leqq C\left(n, \lambda_{N}(\infty)\right) e^{-\omega(T+m-1)} \lambda_{N}(\infty) \rightarrow 0
$$

as $m \rightarrow \infty$, which means that $\left\{u^{m}\right\}$ is a Cauchy sequence in $E_{N-1}$. Therefore, there exists a limit $u \in E_{N-1}(\mathbb{R})$ such that

$$
\lim _{m \rightarrow \infty} \max _{T_{1} \leqq t \leqq T_{2}}\left\|\bar{\partial}^{N-2}\left(U^{m}(t, \cdot)-U(t, \cdot)\right)\right\|=0
$$

for any $-\infty<T_{1}<T_{2}<\infty$. From (6.1), (6.2), (6.3), (6.12) and the fact that $N-2 \geqq[n / 2]+1$, we see easily that

$$
\begin{gathered}
P(u) u=f \quad \text { in } \mathbb{R} \times \Omega \text { and } u=0 \text { on } \mathbb{R} \times \partial \Omega, \\
\|U(t, \cdot)\|_{\infty} \leqq K / 2 \quad \text { for } t \in \mathbb{R}, \\
\left\|\bar{\partial}^{k} U(t, \cdot)\right\| \leqq C(k) \lambda_{k}(\infty) \quad \text { for } t \in \mathbb{R} \text { and } 0 \leqq k \leqq N-2 .
\end{gathered}
$$

Now, let us prove that $u \in E_{N}(\mathbb{R})$, using our local existence theorem: Theorem 5.1. In order to do that, first we shall show that

$$
\bar{\partial}_{t}^{j+1} u(t, \cdot) \in H^{N-j} \cap H_{0}^{1} \quad \text { and } \quad\left\|\bar{\partial}_{t}^{j+1} u(t, \cdot)\right\|_{N-j} \leqq C(N) \lambda_{N}(\infty)
$$

for any $j \in[0, N-2]$ and $t \in \mathbb{R}$. For any $\phi \in C_{0}^{\infty}(\Omega)$ and multi-index $\alpha$ with $|\alpha|=N-j$, we have

$$
\left|\left(\partial_{x}^{\alpha} \bar{\partial}_{t}^{j+1} u(t, \cdot), \phi\right)\right| \leqq\left\|\bar{\partial}_{t}^{j+1}\left(u(t, \cdot)-u^{m}(t, \cdot)\right)\right\|\left\|\partial_{x}^{\alpha} \phi\right\|+C(n) \lambda_{N}(\infty)\|\phi\|
$$

for any $m$. Combining this and (6.12) implies that $\bar{\partial}_{t}^{j+1} u(t, \cdot) \in H^{N-j}$ and $\left\|\bar{\partial}_{t}^{j+1} u(t, \cdot)\right\|_{N-j} \leqq C(N) \lambda_{N}(\infty)$, because $C_{0}^{\infty}(\Omega)$ is dense in $L^{2}$. For any $\varepsilon>0$ and $\phi \in H_{0}^{1}$, there exists a $\psi \in C_{0}^{\infty}(\Omega)$ such that $\|\phi-\psi\|_{1}<\varepsilon$. And then, we have

$$
\begin{array}{r}
\lim _{m, m^{\prime} \rightarrow \infty}\left|\left(\bar{\partial}_{t}^{j+1}\left(u^{m}-u^{m^{\prime}}\right)(t, \cdot), \phi\right)_{1}\right| \leqq \lim _{m, m^{\prime} \rightarrow \infty}\left\{\left\|\bar{\partial}_{t}^{j+1}\left(u^{m}-u^{m^{\prime}}\right)(t, \cdot)\right\|_{1}\|\phi-\psi\|_{1}\right. \\
\left.+\sum_{|\alpha|=1}\left|(-1)^{\alpha}\left(\bar{\partial}_{t}^{j+1}\left(u^{m}-u^{m^{\prime}}\right)(t, \cdot), \partial_{x}^{2 \alpha} \psi\right)\right|\right\} \leqq 2 \lambda_{N}(\infty) \varepsilon,
\end{array}
$$

which implies that $\left\{\bar{\partial}_{t}^{j+1} u^{m}(t, \cdot)\right\}$ is a Cauchy sequence in the weak topology of $H_{0}^{1}$. Since $\bar{\partial}_{t}^{j+1} u^{m}(t, \cdot)$ converges to $\bar{\partial}_{t}^{j+1} u(t, \cdot)$ in the strong topology of $L^{2}$ for each $t \in \mathbb{R}$ as follows from (6.12), we have $\bar{\partial}_{t}^{j+1} u(t, \cdot) \in H_{0}^{1}$ for each $t \in \mathbb{R}$, which proves (6.16). 
Since we do not know the existence of $\partial_{t}^{N} u(t, \cdot)$ until now, instead of $\partial_{t}^{N}(t, \cdot)$ we consider

$$
\begin{aligned}
u_{N}(t, \cdot)= & A_{0}(U(t, \cdot))^{-1}\left\{A_{i j}(U(t, \cdot)) \partial_{i} \partial_{j} \partial_{t}^{N-2} u(t, \cdot)+B_{i j}(U(t, \cdot)) \partial_{i} \partial_{j} \partial_{t}^{N-1} u(t, \cdot)\right. \\
& \left.-A_{j}(U(t, \cdot)) \partial_{j} \partial_{t}^{N-1} u(t, \cdot)+\partial_{t}^{N-2} f(t, \cdot)+\left[\partial_{t}^{N-2}, P(u)\right] u(t, \cdot)\right\} \cdot(6.17)
\end{aligned}
$$

Using Lemmas 3.1.1 and 3.1.2-(2) and (6.12), we see that $\left[\partial_{t}^{N-2}, P\left(u^{m}\right)\right] u^{m}(t, \cdot)$ converges to $\left[\partial_{t}^{N-2}, P(u)\right] u(t, \cdot)$ strongly in $L^{2}$ for each $t \in \mathbb{R}$ as $m \rightarrow \infty$. Therefore, employing the same arguments as before, from (6.12) and the second part of (6.16) we see that $\partial_{t}^{N} u^{m}(t, \cdot)$ converges to $u_{N}(t, \cdot)$ weakly in $L^{2}$ and $\left\{\partial_{t}^{N} u^{m}(t, \cdot)\right\}$ is a Cauchy sequence in the weak topology of $H_{0}^{1}$ for each $t \in \mathbb{R}$, which implies that

$$
u_{N}(t, \cdot) \in H_{0}^{1} \text { and }\left\|u_{N}(t, \cdot)\right\| \leqq C(n) \lambda_{N}(\infty) .
$$

For any $T \in \mathbb{R}$, let us consider the following initial boundary value problem:

$$
\begin{gathered}
P(v) v=f \quad \text { in } \quad\left[T, T+T_{1}\right] \times \Omega, \quad v=0 \quad \text { on }\left[T, T+T_{1}\right] \times \partial \Omega, \\
v(T, \cdot)=u(T, \cdot) \text { and } v_{t}(T, \cdot)=u_{t}(T, \cdot) \quad \text { in } \Omega .
\end{gathered}
$$

In view of (6.16) and (6.18), the compatibility condition for (6.19) is satisfied and then Theorem 5.1 implies that there exists a $T_{1}>0$ independent of $T$ such that (6.19) admits a unique solution $v \in E_{N}\left(\left[T, T+T_{1}\right]\right)$ satisfying the condition: $\|V(t, \cdot)\|_{\infty} \leqq 2 K / 3$ for $t \in\left[T, T+T_{1}\right]$. Applying (3.2.3) in the proof of Theorem 3.2.1 to the problem:

$$
\begin{aligned}
P(v)(v-u) & =(P(u)-P(v)) u \quad \text { in }\left[T, T+T_{1}\right] \times \Omega, \\
v-u & =0 \quad \text { on }\left[T, T+T_{1}\right] \times \partial \Omega, \\
(v-u)(T, x) & =(v-u)_{t}(T, x)=0 \quad \text { in } \Omega,
\end{aligned}
$$

and using (6.15) and Sobolev's imbedding theorem, we have

$$
\|(V-U)(t, \cdot)\| \leqq C \int_{T}^{t}\left(1+p_{v}(s)+p_{v}(s)^{2}\right)\|(V-U)(s, \cdot)\|^{2} d s
$$

for any $t \in\left[T, T+T_{1}\right]$. Since $v(t, \cdot) \in E_{N}\left(\left[T, T+T_{1}\right]\right), p_{v}(s)$ is bounded in $\left[T, T+T_{1}\right]$. And then, application of Gronwall's inequality to (6.20) implies that $\|U(t, \cdot)-V(t, \cdot)\|=0$ for $t \in\left[T, T+T_{1}\right]$. Combining this and Poincaré's inequality, we see that $u(t, \cdot)=v(t, \cdot) \in E_{N}\left(\left[T, T+T_{1}\right]\right)$. Since $T$ is chosen arbitrarily and since $T_{1}$ is independent of $T$, we have $u \in E_{N}(\mathbb{R})$.

Finally, we shall show that $u\left(t+T_{0}, \cdot\right)=u(t, \cdot)$ for any $t \in \mathbb{R}$. Put $v(t, x)=u\left(t+T_{0}, \cdot\right)$, and then $P(u)(v-u)=(P(u)-P(v)) v$ in $\mathbb{R} \times \Omega$ and $u-v=0$ on $\mathbb{R} \times \partial \Omega$. Since $\|(P(u)-P(v)) v\| \leqq C \lambda_{N_{0}}(\infty)\|V-U\|$, applying Theorem 3.2.1, we have for $t>S$,

$$
\begin{gathered}
e^{2 \omega t}\|(U-V)(t, \cdot)\|^{2}+\int_{S}^{t} e^{2 \omega s}\|(U-V)(s, \cdot)\|^{2} d s \\
\leqq C\left\{e^{2 \omega S}\|(U-V)(S, \cdot)\|^{2}+\lambda_{N_{0}}(\infty)^{2} \int_{S}^{t} e^{2 \omega s}\|(U-V)(s, \cdot)\|^{2} d s\right\} .
\end{gathered}
$$


Choosing $\lambda_{N_{0}}(\infty)$ so small that $C \lambda_{N_{0}}(\infty)^{2} \leqq 1$, we have

$$
\|(U-V)(t, \cdot)\| \leqq C e^{-\omega(t-S)}\|(U-V)(S, \cdot)\| \leqq 2 C \lambda_{N_{0}}(\infty) e^{-\omega(t-S)}
$$

for any $t>S$. Tending $S$ to $-\infty$, we have $\|(U-V)(t, \cdot)\|=0$ for any $t \in \mathbb{R}$. Combining this and Poincaré's inequality implies that $u=v$, that is, $u(t, \cdot)=u\left(t+T_{0}, \cdot\right)$ for any $t \in \mathbb{R}$. This completes the proof of the assertion (A) of Theorem 2.3.

Employing the same argument, by Theorem 3.2.1 we have the assertion (2) of Theorem 2.3. In the same manner as in proving (6.10), we see the assertion (3) of Theorem 2.3. Therefore, we may finish the proof of Theorem 2.3.

\section{References}

1. Andrews, G.: On the existence of solutions to the equation $u_{t t}=u_{x x t}+\sigma\left(u_{x}\right)_{x}$. J. Diff. Eq. 35, 200-231 (1980)

2. Andrews, G., Ball, J.M.: Asymptotic behaviour and changes in phase in one-dimensional nonlinear viscoelasticity. J. Diff. Eq. 44, 306-341 (1982)

3. Ang, D.D., Dinh, A.P.N.: On the strongly damped wave equation: $u_{t t}-\Delta u-\Delta u_{t}+f(u)=0$. SIAM J. Math. Anal. 19, 1409-1418 (1988)

4. Arima, R., Hasegawa, Y.: On global solutions for mixed problem of semilinear differential equation. Proc. Jpn Acad. 39, 721-725 (1963)

5. Aviles, P., Sandefur, J.: Nonlinear second order equations with applications to partial differential equations. J. Diff. Eq. 58, 404-427 (1985)

6. Cleménts, J.: Existence theorems for a quasilinear evolution equation. SIAM J. Appl. Math. 26, 745-752 (1974)

7. Cleménts, J.: On the existence and uniqueness of solutions of the equation $u_{t t}-$ $\left(\partial / \partial x_{i}\right) \sigma_{i}\left(u_{x_{i}}\right)-\Delta_{N} u_{t}=f$. Canad. Math. Bull. 18, 181-187 (1975)

8. Dafermos, C.M.: The mixed initial-boundary value problem for the equations of nonlinear one-dimensional visco-elasticity. J. Diff. Eq. 6, 71-86 (1969)

9. Davis, P.: A quasi-linear hyperbolic and related third order equation. J. Math. Anal. Appl. 51, 596-606 (1975)

10. Ebihara, Y.: On some nonlinear evolution equations with the strong dissipation. J. Diff. Eq. 30, 149-164 (1978); II, ibid 34, 339-352 (1979); III, ibid 45, 332-355 (1982)

11. Ebihara, Y.: Some evolution equations with the quasi-linear strong dissipation. J. Math. Pures et Appl. 58, 229-245 (1979)

12. Engler, H.: Strong solutions for strongly damped quasilinear wave equations. Contemp. Math. 64, 219-237 (1987)

13. Friedman, A., Necas, J.: Systems of nonlinear wave equations with nonlinear viscosity. Pacific J. Math. 135, 29-55 (1988)

14. Greenberg, J.M., MacCamy, R.C., Mizel, J.J.: On the existence, uniqueness, and stability of the equation $\sigma^{\prime}\left(u_{x}\right) u_{x x}-\lambda u_{x x t}=\rho_{0} u_{t t}$. J. Math. Mech. 17, 707-728 (1968)

15. Greenberg, J.M.: On the existence, uniqueness, and stability of the equation $\rho_{0} X_{t t}=E\left(X_{x}\right) X_{x x}+\lambda X_{x x t}$. J. Math. Anal. Appl. 25, 575-591 (1969)

16. Kato, T.: Abstract differential equations and nonlinear mixed problem. Scuola Normale Superiore, Lezioni Fermiane, Pisa (1985)

17. Matsumura, A.: Global existence and asymptotics of the solutions of the second-order quasilinear hyperbolic equations with the first-order dissipation. Publ. RIMS, Kyoto Univ. 13, 349-379 (1977)

18. Mizohata, K., Ukai, S.: The global existence of small amplitude solutions to the nonlinear acoustic wave equation. Preprint in 1991, Department of Information Sci., Tokyo Inst. of Tech.

19. Pecher, H.: On global regular solutions of third order partial differential equations. J. Math. Anal. Appl. 73, 278-299 (1980)

20. Potier-Ferry, M.: On the mathematical foundation of elastic stability, I. Arch. Rational Mech. Anal. 78, 55-72 (1982) 
21. Rabinowitz, P.: Periodic solutions of nonlinear partial differential equations. Commun. Pure Appl. Math. 20, 145-205 (1967); II, ibid 22, 15-39 (1969)

22. Shibata, Y.: On the Neumann problem for some linear hyperbolic systems of $2^{\text {nd }}$ order with coefficients in Sobolev spaces. Tsukuba J. Math. 13, 283-352 (1989)

23. Shibata, Y., Kikuchi, M.: On the mixed problem for some quasilinear hyperbolic system with fully nonlinear boundary condition. J. Diff. Eq. 80, 154-197 (1989)

24. Webb, G.F.: Existence and asymptotic behavior for a strongly damped nonlinear wave equation. Canada J. Math. 32, 631-643 (1980)

25. Yamada, Y.: Some remarks on the equation $y_{t t}-\sigma\left(y_{x}\right) y_{x x}-y_{x t x}=f$. Osaka J. Math. 17, 303-323 (1980)

Communicated by H. Araki 